\title{
Cobertura de TV Digital: Avaliação Subjetiva e Medições Em um Cenário Real
}

\author{
Aureliano Magalhães de S. Neto, A. B. de F. Diniz, M. E. C. Rodrigues, V. A. de Sousa Jr., H. B. Mendonça
}

\begin{abstract}
Open and free Digital TV remains an important and popular service in Latin America, specially in Brazil with the implantation of the Brazilian Digital Terrestrial Television System (SBTVD-T). This paper presents a case study of power measurements obtained according to the ABNT 15604/2017 standard, aiming to compare the coverage area of the digital television service in the city of Natal with that projected with SIGAnatel, which runs the point-to-area prediction method ITU-R P.1546. As a main result, we intended to fill the gap with technical material related to the discussion of digital TV in Brazil by presenting measures of power from stations in operation as well as subjective signal quality evaluations provided by final users for comparison purposes.
\end{abstract}

\section{Palavras-Chave-Digital TV, Measurement.}

\section{INTRODUÇÃo}

A medição de sinais em ambientes externos é uma atividade frequente e de inestimável valor no campo das telecomunicações, sendo realizada para diversos propósitos, como prover análise da qualidade de serviços prestados ao usuário e fiscalizar se as legislações dos mais variados serviços de telecomunicações estão sendo, de fato, cumpridas.

Em termos de fiscalização, o que a legislação brasileira [1] estabelece como sendo um dos requisitos para a execução do serviço de radiodifusão de sons e imagens é o atendimento de, no mínimo, $90 \%$ da população residente no município que a emissora detém outorga, comprovação esta observada por meio da análise de projeto técnico. Na prática, a medição de sinais e a análise subjetiva dos usuários são fundamentais para identificar regiões de má cobertura dentro do contorno de serviço e auxiliam na compreensão dos fatores contribuintes.

Além da já apresentada preocupação com a área de cobertura, existe o cuidado para que se garanta a mínima distância segura entre o local de instalação do sistema irradiante e as áreas residenciais, com vistas a garantir que a intensidade e a exposição à radiação não ionizante (RNI) estejam dentro dos valores aceitáveis conforme estabelecido na Resolução n 700, da Anatel, de 28 de setembro de 2018.

Com o intuito de facilitar a adequação do projeto técnico às legislações que compreendem o serviço de radiodifusão de sons e imagens, a Agência Nacional de Telecomunicações (Anatel) disponibiliza, em sua página eletrônica, ferramentas computacionais e modelos de documentos que servem como guia para a adequação do projeto técnico às normas vigentes. Contudo, é relevante mencionar que habitualmente

Os autores Aureliano M. de S. Neto, André B. de F. Diniz, Marcio E. C. Rodrigues e Vicente A. de Sousa Jr. são da Universidade Federal do Rio Grande do Norte (UFRN), Natal, RN. E-mails: aureliano.magalhaes@gmail.com, andrebfd@ufrn.edu.br, marcio.rodrigues@ct.ufrn.br, vicente.sousa@ufrn.edu.br. O autor Halysson B. Mendonça é da Agência Nacional de Telecomunicações, Natal, RN, Brazil, E-mail: halysson@anatel.gov.br. Este trabalho foi realizado com apoio da Coordenação de Aperfeiçoamento de Pessoal de Nível Superior - Brasil (CAPES) - Código de Financiamento 001. há diferenças entre os modelos computacionais e as áreas reais de cobertura, sobretudo decorrentes de construções capazes de afetar consideravelmente a propagação multi-percurso.

Este trabalho se destina a apresentar um caso de estudo de medições de sinais, tendo como alvo sete emissoras de televisão digital na cidade de Natal/RN e com pontos de medição escolhidos com base na aferição subjetiva da percepção dos telespectadores em termos de qualidade da recepção em suas residências.

\section{A. Trabalhos Relacionados}

$\mathrm{Na}$ comunidade científica é notada a preocupação existente com relação à qualidade da recepção de sinais dos sistemas de televisão digital, evidenciada através de estudos voltados para a área de dispositivos IEEE 802.11p sem licença [2], IEEE 802.22 WRAN, redes DVB-T2 [3], sistemas legados de comunicação móvel [4] e sistemas 5G [5], sendo também muito comuns os estudos cuja finalidade é avaliação da interferência entre canais adjacentes, tanto para a tecnologia analógica, quanto digital [6] e a medição de interferência nas proximidades de uma antena receptora de televisão digital [7].

Contudo, a quantidade de trabalhos científicos desenvolvidos com vistas a avaliar regiões de cobertura dos serviços de radiodifusão de sons e imagens com tecnologia digital e a qualidade de recepção desses serviços na residência dos telespectadores é demasiadamente escassa no Brasil, sendo este o primeiro trabalho científico desenvolvido na cidade de Natal/RN com esta finalidade.

Este estudo visa preencher essa lacuna, trazendo dados de medições realizadas em campo para caracterizar a situação do atendimento de serviço para um número relevante de clientes. Em vista da problemática discutida, as contribuições principais deste estudo são: (i) Apresentar a percepção dos telespectadores na cidade de Natal/RN em relação a qualidade dos serviços disponibilizados por meio da análise de dados obtidos através de questionário respondido por uma amostra da população; (ii) Descrever o setup de medição, o processo utilizado e denotar os locais onde a atividade foi realizada; e (iii) Confrontar os dados oriundos das medições com as respostas obtidas por meio do questionário supracitado e com a legislação vigente, comparando dados quantitativos (medidas de sinal) e dados subjetivos, frutos da pesquisa de satisfação.

Este trabalho encontra-se estruturado da seguinte forma: a Seção II aborda aspectos regulatórios relacionados à prestação do serviço de televisão digital no Brasil, aspectos estes baseados em recomendações internacionais e normativas nacionais; a Seção III descreve as medições de sinais realizadas em Natal/RN e o setup utilizado no experimento, sendo os resultados obtidos apresentados e discutidos na Seção IV; e a Seção V finaliza o artigo com as conclusões acerca do trabalho realizado. 


\section{Aspectos RegulatóRios}

As normas de implantação de sistemas de televisão digital no Brasil são elaboradas pela Anatel, seguindo recomendações da International Telecommunications Union (ITU) no que se refere à metodologia utilizada para determinação da área de cobertura de cada entidade responsável pela transmissão e/ou retransmissão do serviço de radiodifusão. As normas técnicas da Agência Brasileira de Normas Técnicas (ABNT) são utilizadas como referencial do ponto de vista da recepção desse serviço, a partir do momento em que torna possível estabelecer os requisitos mínimos através dos quais os receptores de televisão digital são capazes de sintonizar os canais das emissoras atuantes na região de estudo.

Em relação aos serviços de radiodifusão de sons e imagens, a medição de sinais é importante por dois principais motivos: i) possibilita às emissoras analisarem a cobertura na região de atuação e identificar possíveis problemas e/ou estudar intervenções capazes de melhorar a qualidade do serviço prestado, e ii) possibilita às entidades reguladoras avaliarem se cada emissora está operando dentro dos limites e áreas de abrangência de acordo com o Plano Básico de Televisão Digital (PBTVD). O segundo motivo é fiscalizar se o serviço prestado por uma dada emissora está, de fato, limitado à área (região, município) de outorga objetivando minimizar a ocorrência de problemas possivelmente ocasionados, por exemplo, pela interferência entre canais utilizados por emissoras em municípios próximos. O segundo motivo também possibilita determinar se a potência de operação do sistema de transmissão e/ou retransmissão de uma dada emissora é, de fato, suficiente para assegurar uma boa cobertura na região de outorga com base no que é estabelecido pela legislação do serviço de radiodifusão de sons e imagens.

De acordo com o no Art. 16 da Portaria n ${ }^{\circ}$ 925, de 22 de agosto de 2014, "a cobertura deve atender necessariamente a, no mínimo, $90 \%$ da área urbana do município objeto do ato de outorga, conforme a base de dados dos setores censitários mais recente do Instituto Brasileiro de Geografia e Estatística - IBGE, obedecendo as características previstas para o canal no PBTVD”. Para os canais de televisão digital, o contorno de serviço delimita a região cuja intensidade de campo elétrico é maior ou igual a $51 \mathrm{~dB} \mu \mathrm{V} / \mathrm{m}$ [8], tomando-se como referência a localização geográfica do sistema irradiante da estação, e corresponde, segundo a Portaria $n^{\circ} 925$, de 22 de agosto de 2014, ao "lugar geométrico dos pontos onde a intensidade de campo é excedida em $50 \%$ dos locais e em $90 \%$ do tempo, segundo o método de predição de propagação ponto-área estabelecido no Regulamento Técnico para Prestação dos Serviços de Radiodifusão de Sons e Imagens e de Retransmissão de Televisão, considerando a antena receptora situada a 10 metros de altura".

A Recomendação da ITU, embora seja norma mais conhecida e utilizada pela sua riqueza de detalhes que possibilitam a utilização de antena receptora situada em alturas menores por meio do emprego de métodos de interpolação, não é a única norma capaz ser utilizada para avaliação da área de cobertura dos sistemas de televisão digital no Brasil. A norma ABNT 15604, de 19 de outubro de 2017, também é amplamente utilizada como referência e especifica o conjunto de funcionalidades essenciais requeridas dos dispositivos de recepção de televisão digital de 13 segmentos (full-seg), assim como os de um segmento (one-seg), destinados a receber sinais na modalidade fixa, móvel e portátil".

Dentre os aspectos abordados por essa norma, são descritos os critérios de sensibilidade necessários para que o receptor possa, a partir do sinal captado por antena interna ou externa, reproduzir o sinal de televisão digital com qualidade e de forma ininterrupta. Com base nas especificações técnicas dos sistemas de transmissão das emissoras de televisão atuantes na cidade de Natal/RN, dados estes obtidos no site da Anatel, é possível estimar, com boa precisão, a área de cobertura das emissoras de televisão digital através do Sistema de Informações Geográficas da Anatel (SIGAnatel), área esta gerada pela plataforma que utiliza como referência a própria recomendação ITU-R P.1546.

O que se realiza neste artigo é a comparação dos dois modelos por meio da medição de sinais utilizando os valores de referência para os receptores de televisão digital com base nas especificações da norma ABNT 15604 [9]. Ela considera $-77 \mathrm{dBm}$ como nível mínimo de potência de recepção sinais, dentro de uma área de cobertura gerada no SIGAnatel.Por fim e com base nesse valor de referência, é possível demarcar a região mais propícia para o bom funcionamento da televisão digital, estabelecendo o que pode ser caracterizado como sendo a área real de atendimento desse serviço na cidade de Natal/RN.

\section{Medições: Material e Procedimentos}

Esta seção apresenta o setup experimental usado para medições em campo, bem como as emissoras de televisão que foram avaliadas neste estudo de caso. Posteriormente são apresentados os resultados obtidos da análise qualitativa via questionário e os locais alvo de estudo. A referida seção é concluída com a apresentação do cronograma de realização da atividade e uma breve explicação do protocolo de medição utilizado.

\section{A. Setup Experimental e Estações Estudadas}

Para as medições realizadas neste trabalho, foram utilizados os seguintes equipamentos:

- Analisador de espectro Advantest U3641, com faixa de operação de $9 \mathrm{kHz}$ a $3 \mathrm{GHz}$;

- Antena log-periódica Rohde \& Schwarz modelo HL223, faixa de operação de $200 \mathrm{MHz}$ a $1,3 \mathrm{GHz}$;

- Mastro telescópico de 1,70 m de altura;

- Cabo coaxial Huber+Suhner RG58 com 3 metros;

- Veículo automotor utilizado para o deslocamento entre os variados pontos de medição; e

- Inversor veicular para energizar o analisador de espectro durante a atividade de medição.

$\mathrm{O}$ analisador de espectro Advantest U3641 permaneceu conectado ao inversor veicular (tomada automotiva 12V), permitindo assim o seu funcionamento durante o processo de medição de sinais em diferentes locais da cidade. A antena, devidamente posicionada no topo do mastro telescópico, foi conectada ao analisador de espectro por meio do cabo coaxial. A Fig. 1 ilustra o processo de medição na prática e a Fig. 2 representa o diagrama de blocos do setup utilizado para a realização da atividade.

A antena utilizada apresenta características adequadas para a recepção de televisão digital tanto no que se refere à faixa de 


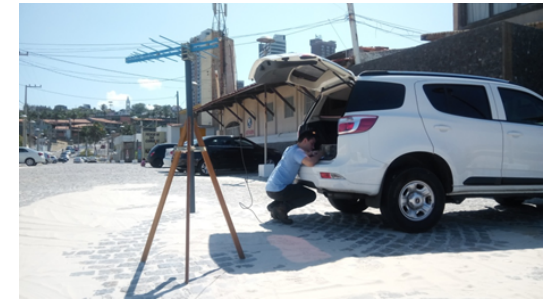

Fig. 1. Aparato utilizado nas medições.

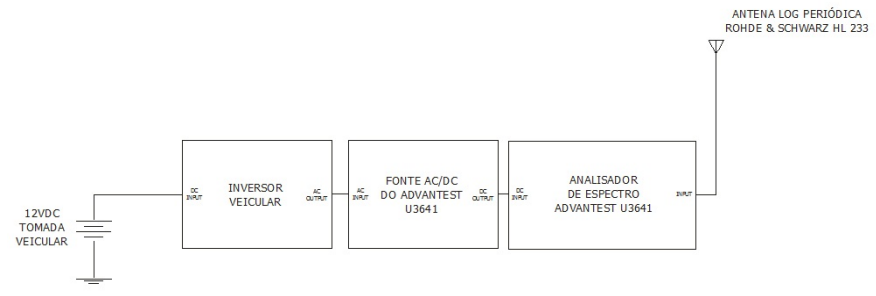

Fig. 2. Diagrama de blocos do setup de medição.

operação (UHF), quanto à forma do seu diagrama de radiação, que pode ser encontrado em [10].

As emissoras que foram alvo do estudo de caso deste artigo são mostradas na Tabela I [11].

TABELA I

EMISSORAS ALVO DO ESTUDO DE CASO.

\begin{tabular}{lllll}
\hline \hline \multirow{2}{*}{ NOME FANTASIA } & \multirow{2}{*}{ CANAL } & \multicolumn{3}{c}{ FREQUENCIA (MHz) } \\
& & MÍN & CENTRAL & MÁX \\
\hline TV FELIZ & 16 & 482 & 485 & 488 \\
INTERTV CABUGI & 34 & 590 & 593 & 596 \\
TV PONTA NEGRA & 36 & 602 & 605 & 608 \\
TV BANDEIRANTES & 30 & 566 & 569 & 572 \\
TV TROPICAL & 32 & 578 & 581 & 584 \\
TVU & 48 & 674 & 677 & 680 \\
FUNDAÇÃO ANTÔNIO & \multirow{2}{*}{14} & 470 & 473 & 476 \\
GOMES DOS SANTOS & & & & \\
\hline \hline
\end{tabular}

\section{B. Questionário de Avaliação de Qualidade e Definição dos Pontos de Medição}

O questionário sobre a qualidade do serviço oferecido pelas emissoras de TV digital foi realizado eletronicamente pela Internet, sendo divulgado em rede interna da Universidade Federal do Rio Grande do Norte (UFRN) e nas redes sociais mais acessadas. Assim, foi possível contar com a colaboração de indivíduos de outras instituições de ensino e de diferentes regiões da cidade de Natal/RN, totalizando 172 respostas de pessoas de diferentes faixas etárias. O questionário é foi concebido pelos autores deste artigo e é disponibilizado em https://github.com/vicentesousa/projetoTV.

Os entrevistados foram separados por regiões com base em suas respostas (bairros e logradouros), as quais foram marcadas em mapa com auxílio do software Google Earth. Essa informação foi útil para a construção do itinerário e locais de medições e auxiliou na elaboração do cronograma.

Por meio do SIGAnatel, disponibilizado de forma gratuita por meio do endereço eletrônico http://sistemas. anatel.gov.br/siganatel/, é possível acessar as tabelas da recomendação ITU-R 1546 [12] e os contornos das estações geradoras. Por intermédio dessa ferramenta, analisou-se tecnicamente a contribuição de cada estação geradora de televisão digital alvo do estudo. Tal procedimento foi importante para estimar a região atendida por essas estações, delimitando a área de interesse para realização das medições. Todos os dados relativos às estações transmissoras de televisão digital estão disponíveis no sistema MOSAICO da Anatel, podendo ser acessos por meio do endereço: http: //sistemas.anatel.gov.br/se/public/view/b/ srd.php

Após realizar a análise via SIGAnatel sobre as estações, averiguou-se que todas elas possuem condições técnicas teoricamente satisfatórias para atendimento à cidade, área para a qual a equipe possuía recursos (tempo para realização de medições e dados qualitativos dos usuários) para uma análise mais profunda. Tal análise é composta pelas medições descritas a seguir, complementadas pelo resultado do questionário de avaliação de qualidade. Para a definição dos dias e horários de medição levou-se em consideração uma série de fatores, tais como: disponibilidade de veículo automotor, motorista e dos equipamentos que constituem o setup de medição, que foram emprestados pela Anatel. Os pontos analisados são mostrados na Fig. 3.

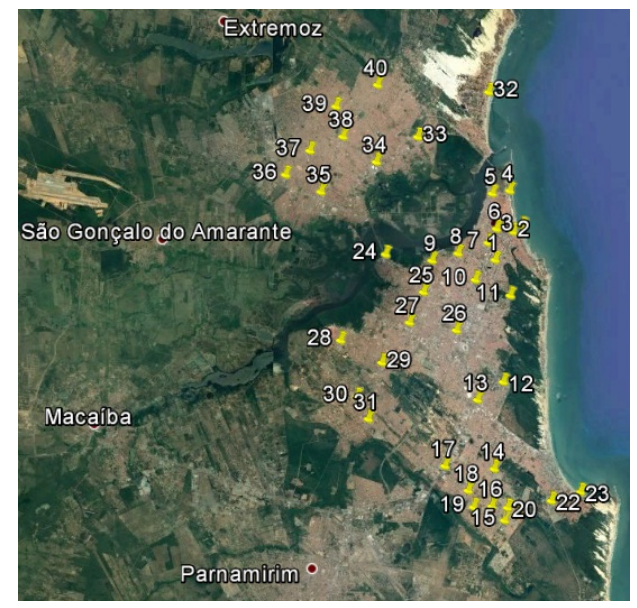

Fig. 3. Localidades analisadas durante o estudo.

A Fig. 4 permite a visualização aproximada de onde os sistemas irradiantes das emissoras de televisão digital estão instaladas na cidade de Natal/RN. As informações de instalação são encontradas na página eletrônica da Anatel.

\section{Procedimento de Medição do Sinal}

As medições foram realizadas em 04 dias, em outubro de 2019 , entre as $13 \mathrm{~h} 30 \mathrm{~min}$ e $16 \mathrm{~h} 30 \mathrm{~min}$, tendo sido realizadas 280 (duzentos e oitenta) medidas em 40 pontos distintos: O cronograma de medição foi:

- Medição 1: 01/10/2019 - pontos 1 a 11;

- Medição 2: 10/10/2019 - pontos 12 a 23;

- Medição 3: 17/10/2019 - ponto 24;

- Medição 4: 24/10/2019 - pontos 25 a 40.

$\mathrm{O}$ estudo foi direcionado às localidades onde se obteve o maior número de reclamações no questionário de satisfação. Na maioria dos casos, essas localidades se tratavam das regiões onde as antenas externas dos entrevistados estavam instaladas a menores alturas em relação ao solo.

O protocolo de medição utilizado neste trabalho foi baseado na norma ABNT 15604/2017 [9], implementado dentro de uma área averiguada via SIGAnatel (de acordo com a norma 1546 da ITU [12]). Por mais que a área de atendimento das 


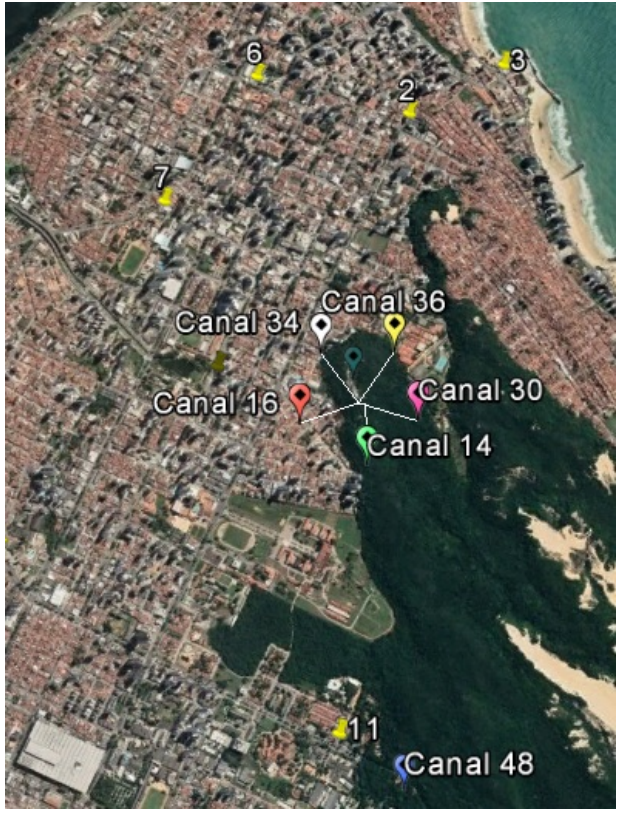

Fig. 4. Localização dos sistemas irradiantes das emissoras de televisão digital. emissoras de televisão digital se estenda até municípios mais distantes, abrangendo, deste modo, a região metropolitana do Rio Grande do Norte (via Recomendação ITU-R P. 1546 através do contorno de serviço via SIGAnatel), o ponto de interesse aqui é obter indícios que constatem que toda a área da cidade do Natal está sendo bem atendida pelo serviço de televisão digital, o que se propõe verificar por meio das medições.

Não há, entretanto, especificações acerca da altura da antena para as medições; dessa maneira, estabeleceu-se uma distância-padrão de 1,70 m para o solo em todas as medições realizadas. Chegando aos pontos escolhidos para medição (mostrados na Fig. 3), todo o sistema era retirado do veículo automotor e montado. Inicialmente, uma busca era feita por meio da rotação da antena em torno do mastro, pelo azimute no qual eram obtidas as maiores potências de recepção para cada uma das emissoras. A ideia era buscar reproduzir o mesmo procedimento que o morador faz em sua residência ao instalar uma antena: procurar a posição em que a qualidade de recepção seja a melhor possível.

\section{Resultados E Discuss Ão}

A potência em cada ponto de medição está disposta na Tabela II, enquanto o resultado da entrevista (percepção da qualidade de sinal de acordo com a resposta dos entrevistados) é mostrado na Fig. 5. O padrão de planilha usado para o armazenamento das medidas está disponível em https:// github.com/vicentesousa/projetoTV. Os bairros visitados foram os seguintes: Felipe Camarão, Lagoa Nova, Nossa Senhora de Nazaré, Nova Descoberta, Nova Parnamirim, Pajuçara, Parque dos Coqueiros, Petrópolis, Planalto, Ponta Negra, Potengi, e Quintas.

Excetuando aqueles que não souberam opinar, a análise da resposta ao formulário de percepção da qualidade de sinal mostra que todas as emissoras apresentaram resultado, no mínimo, razoável. Os números são: $89,76 \%$ para a Band TV; $88,88 \%$ para a TV Ponta Negra; $85,83 \%$ para a TV Tropical; $85,33 \%$ para a Inter TV Cabugi; $74,69 \%$ para a TV Futuro; $70 \%$ para a TVU; e $60,60 \%$ para a TV Feliz.
TABELA II

PotênCIA EM DBM Nos Pontos DE MEdiçÃo.

\begin{tabular}{|c|c|c|c|c|c|c|c|}
\hline Ponto & $\mathrm{CH}$ & $\mathrm{CH}$ & $\mathrm{CH}$ & $\mathrm{CH}$ & $\mathrm{CH}$ & $\mathrm{CH}$ & $\mathrm{CH}$ \\
\hline Medido & 14 & 16 & 30 & 32 & 34 & 36 & 48 \\
\hline 1 & -51 & -53 & -48 & -47 & -50 & -37 & -49 \\
\hline 2 & -56 & -73 & -56 & -48 & -60 & -56 & -61 \\
\hline 3 & -62 & -67 & -58 & -58 & -63 & -54 & -72 \\
\hline 4 & -60 & -74 & -66 & -62 & -67 & -70 & -72 \\
\hline 5 & -70 & -73 & -63 & -67 & -66 & -63 & -74 \\
\hline 6 & -52 & -67 & -49 & -40 & -54 & -49 & -56 \\
\hline 7 & -46 & -66 & -47 & -45 & -50 & -43 & -43 \\
\hline 8 & -55 & -70 & -60 & -54 & -58 & -56 & -51 \\
\hline 9 & -72 & -75 & -68 & -69 & -69 & -69 & -75 \\
\hline 10 & -54 & -66 & -56 & -46 & -55 & -45 & -62 \\
\hline 11 & -45 & -66 & -54 & -60 & -56 & -57 & -45 \\
\hline 12 & -64 & -73 & -61 & -61 & -62 & -66 & -67 \\
\hline 13 & -68 & -63 & -56 & -54 & -57 & -58 & -53 \\
\hline 14 & -64 & -73 & -64 & -64 & -66 & -69 & -73 \\
\hline 15 & -71 & -75 & -70 & -63 & -66 & -68 & -70 \\
\hline 16 & -75 & -76 & -78 & -75 & -79 & -76 & -80 \\
\hline 17 & -66 & -74 & -66 & -67 & -70 & -70 & -69 \\
\hline 18 & -69 & -76 & -72 & -70 & -66 & -72 & -75 \\
\hline 19 & -75 & -75 & -75 & -75 & -75 & -75 & -75 \\
\hline 20 & -76 & -76 & -76 & -73 & -73 & -74 & -75 \\
\hline 21 & -75 & -75 & -70 & -73 & -73 & -75 & -75 \\
\hline 22 & -71 & -75 & -68 & -70 & -70 & -75 & -75 \\
\hline 23 & -61 & -75 & -51 & -59 & -54 & -58 & -62 \\
\hline 24 & -55 & -66 & -56 & -55 & -62 & -56 & -64 \\
\hline 25 & -49 & -66 & -52 & -61 & -57 & -60 & -61 \\
\hline 26 & -62 & -75 & -67 & -63 & -64 & -67 & -70 \\
\hline 27 & -51 & -61 & -59 & -46 & -55 & -51 & -60 \\
\hline 28 & -63 & -71 & -54 & -57 & -57 & -57 & -60 \\
\hline 29 & -64 & -72 & -72 & -70 & -69 & -71 & -75 \\
\hline 30 & -68 & -75 & -75 & -75 & -75 & -75 & -75 \\
\hline 31 & -75 & -75 & -75 & -75 & -75 & -75 & -75 \\
\hline 32 & -51 & -70 & -57 & -52 & -59 & -47 & -63 \\
\hline 33 & -52 & -83 & -50 & -52 & -53 & -55 & -57 \\
\hline 34 & -73 & -74 & -73 & -73 & -72 & -73 & -74 \\
\hline 35 & -68 & -79 & -67 & -68 & -68 & -69 & -71 \\
\hline 36 & -66 & -76 & -64 & -73 & -65 & -62 & -70 \\
\hline 37 & -51 & -74 & -55 & -60 & -55 & -61 & -60 \\
\hline 38 & -74 & -76 & -60 & -67 & -62 & -65 & -75 \\
\hline 39 & -73 & -74 & -65 & -61 & -54 & -61 & -65 \\
\hline 40 & -75 & -76 & -75 & -75 & -76 & -76 & -75 \\
\hline
\end{tabular}

Ainda, a porcentagem dos consultados que não souberam responder sobre a qualidade de sinal das emissoras foi: $61,62 \%$ para a TV Feliz; $51,74 \%$ para a TV Futuro; $47,67 \%$ para a TVU; 30,23\% para a TV Tropical; 27,90\% para a Band TV; 25,58\% para a Inter TV Cabugi; e 22,51\% para a TV Tropical.

No universo dos 40 pontos investigados, totalizando, deste modo, 280 medições, constatou-se que em 05 medidas não houve cumprimento do item 7.2.5a da norma 15604 da ABNT [9], ou seja, apresentaram potência medida abaixo do valor predito pela norma. No entanto, mesmo com a utilização de um mastro relativamente baixo em comparação ao utilizado pelas emissoras de televisão quando realizam a mesma atividade, os resultados obtidos se mostraram dentro do esperado. É importante mencionar que essas cinco medições (com potência medida inferior a $-77 \mathrm{dBm}$ ) ocorreram em pontos distintos. No ponto 16 (localizado no bairro de Nova Parnamirim), por exemplo, três emissoras apresentaram potência abaixo do limiar previsto em norma: Band TV (-78 dBm), Inter TV Cabugi (-79 dBm) e TVU (-80 dBm).

Vale apontar que esse bairro é uma das regiões em que houve maior número de reclamações quanto à qualidade da recepção de TV digital, havendo, portanto, consenso entre medições e análise qualitativa. Uma vez que a região em torno desse ponto tem crescido demograficamente nos últimos anos, uma hipótese que explicaria o resultado encontrado é a influência na quantidade de novos prédios e construções de 


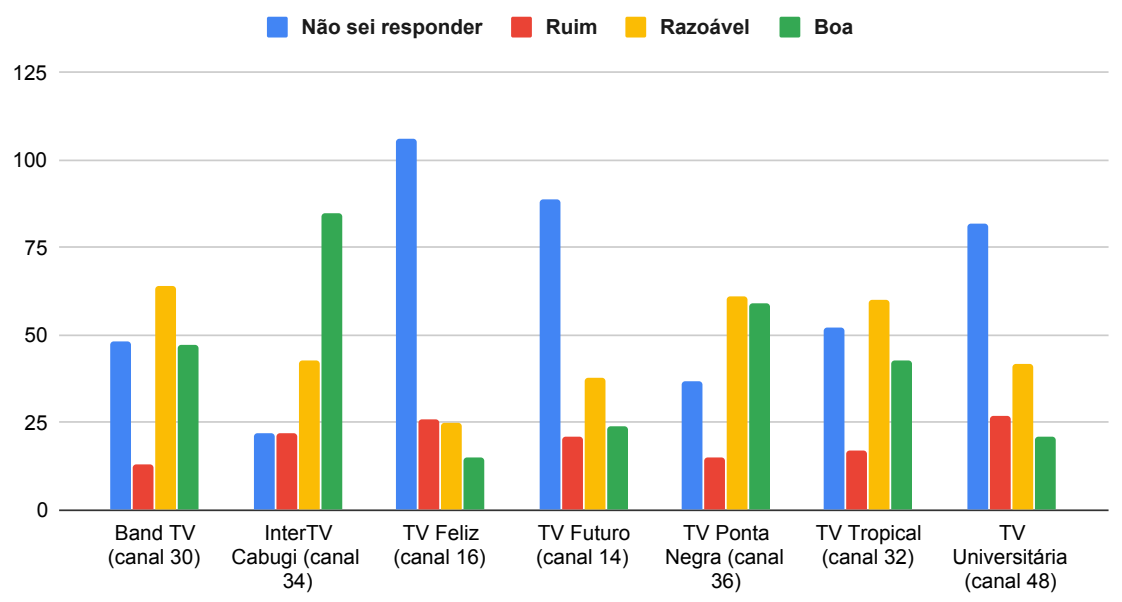

Fig. 5. Percepção dos usuários de televisão digital na Grande Natal.

altura elevada que surgiram na região, podendo comprometer a qualidade da recepção por se tratarem de obstáculos para a propagação multi-percurso. Vale apontar que, embora apenas 03 das 07 estações tenham apresentado potência medida abaixo de $-77 \mathrm{dBm}$, de forma geral, este ponto apresentou desempenho inferior quando comparado aos demais pontos medidos ao longo da cidade de Natal/RN. A medição na referida localização pode ser vista na Fig. 6.

Outros dois locais de medição com potências abaixo de $-77 \mathrm{dBm}$ foram nos bairros Igapó (ponto 33) e Pajuçara (ponto 35), onde os valores encontrados para a emissora TV Feliz foram, respectivamente, $-83 \mathrm{dBm}$ e-79 dBm. Entre a estação transmissora da TV Feliz e os pontos 33 e 35 não foram observados prédios ou demais construções que pudessem servir de obstáculo à propagação multi-percurso, causando degradação considerável no sinal analisado.

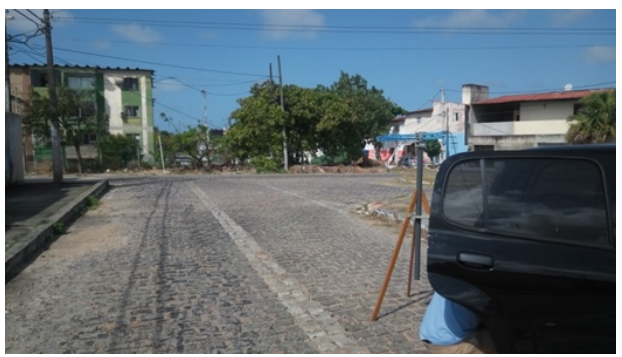

Fig. 6. Medição realizada no ponto 16.

\section{CONClusões}

Este trabalho apresenta um caso de estudo que ilustra um processo de levantamento do atendimento das normas brasileiras na prestação do serviço de TV digital na cidade de Natal. Foram realizadas medições do sinal de sete emissoras de TV. Também foi realizado um questionário online sobre a percepção dos usuários dos serviços de TV.

O projeto técnico para obtenção de outorga do serviço de televisão é baseado em modelos de propagação que muitas vezes não levam em consideração construções e vegetação que podem influenciar a recepção do sinal de TV. Mesmo se o modelo considerar tais aspectos, eles mudam com o tempo (e.g., árvores crescem, prédios são construídos). Assim, realizar medições em campo para verificar as particulares de propagação da região atendida é um procedimento importante para aferição do atendimento das diretivas da Anatel.

Dentre os 40 pontos investigados, em 5 deles não houve cumprimento do item 7.2.5a da norma 15604 da ABNT [9]. Novas medições em tais pontos, com novas condições (e.g., outra altura da antena receptora), são elencadas como trabalhos futuros.

\section{REFERÊNCIAS}

[1] Ministério das Comunicações - GABINETE DO MINISTRO, "Portaria no 925," Diário Oficial da União, 2014, https: //www2.camara.leg.br/comunicacao/rede-legislativa-radio-tv/ arquivos/legislacao-arquivos/portarias-ministerio/ Portarian92522AGO2014AspectostcnicosdaTVdigital.pdf.

[2] M. Fadda, M. Murroni, and V. Popescu, "Interference measurements for unlicensed $802.11 \mathrm{p}$ communication in the TV bands," in 2015 IEEE International Symposium on Broadband Multimedia Systems and Broadcasting, 2015, pp. 1-4.

[3] V. Popescu, M. Fadda, M. Murroni, and D. Giusto, "Coexistence issues for IEEE 802.22 WRAN and DVB-T2 networks," in 2016 IEEE International Symposium on Broadband Multimedia Systems and Broadcasting (BMSB), 2016, pp. 1-4.

[4] A. Guidotti, D. Guiducci, M. Barbiroli, C. Carciofi, P. Grazioso, and G. Riva, "Coexistence and mutual interference between mobile and broadcasting systems," in 2011 IEEE 73rd Vehicular Technology Conference (VTC Spring), 2011, pp. 1-5.

[5] C. Carciofi, A. Garzia, F. Lucidi, and A. Neri, "Coexistence of DVB Television and 5G Services in Adjacent Bands," in 2019 AEIT International Annual Conference (AEIT), 2019, pp. 1-6.

[6] K. Chomsuk and S. Tooprakai, "Estimation on protection distance between analog TV and digital TV in adjacent channel at terrestrial television," in 2017 International Electrical Engineering Congress (iEECON), 2017, pp. 1-4.

[7] V. Petuhov, A. Dvorkovich, and A. Ivchenko, "Determination of the Interfering Signal Field Strength near a TV Receiver," in 2019 International Conference on Engineering and Telecommunication (EnT), 2019, pp. 1-5.

[8] ANATEL, "Agência Nacional de Telecomunicações - CONSELHO DIRETOR - Resolução 583," Diário Oficial da União, 2012.

[9] A. B. de Normas Técnicas, "Norma técnica 15604," 2017.

[10] R. Schwarz, "R\&S HL223 Log-Periodic Antenna - brief description," https://scdn.rohde-schwarz.com/ur/pws/dl_downloads/dl_common_ library/dl_brochures_and_datasheets/pdf_1/HL223_2020.pdf, 2020/2021.

[11] ANATEL, "Agência Nacional de Telecomunicações - Sistema MOSAICO,” 2020, http://sistemas.anatel.gov.br/se/public/view/b/srd. php.

[12] ITU-R, "Recommendation ITU-R P.1546-5: Method for point-to-area predictions for terrestrial services in the frequency range $30 \mathrm{MHz}$ to $3000 \mathrm{MHz}$,' International Telecommunications Union Radiocommunication Sector, 2013. 